\title{
LCLS Strategic Plan
}

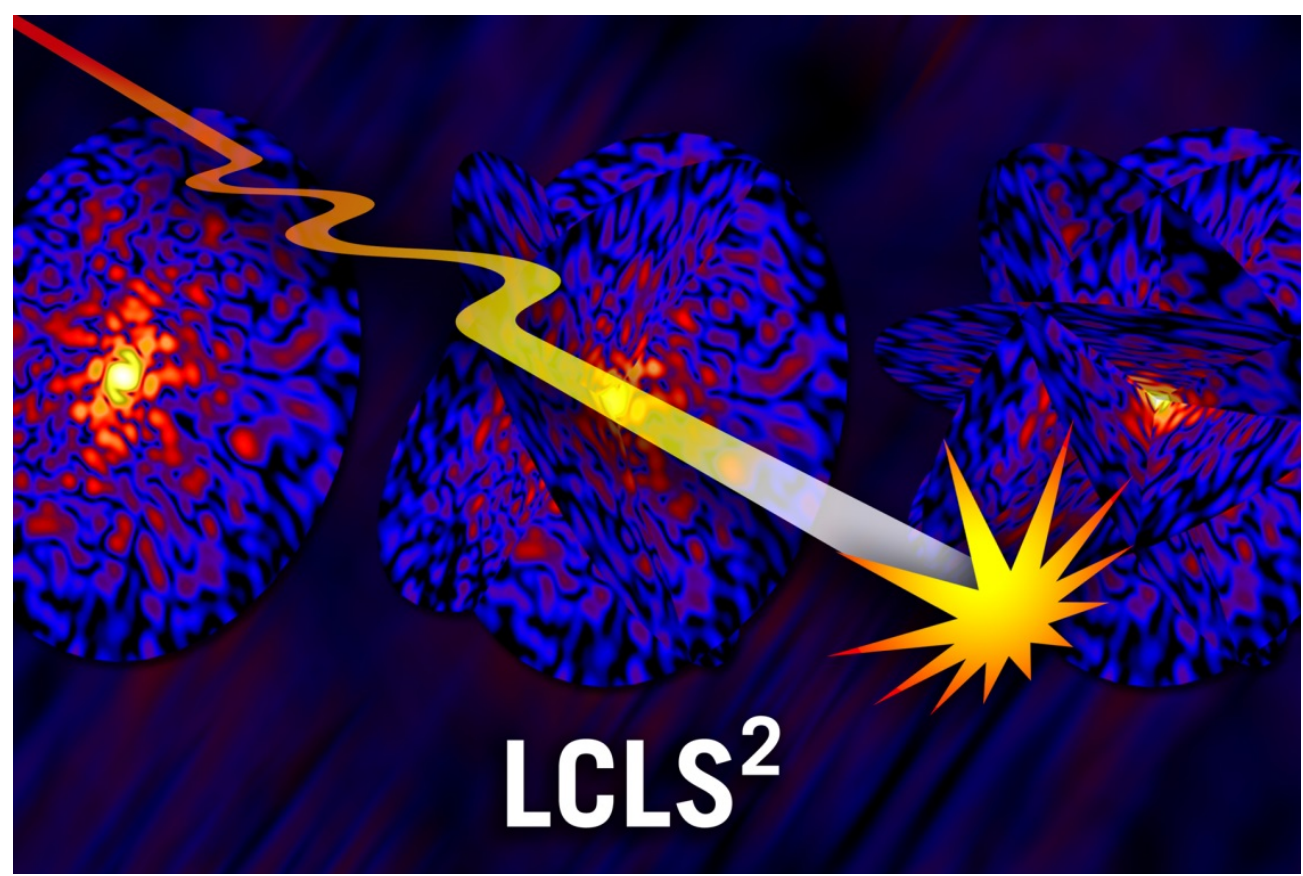

DEPARTMENT OF ENERGY

SLAC NATIONAL ACCELERATOR LABORATORY

April 2013

SLAC-R-1007

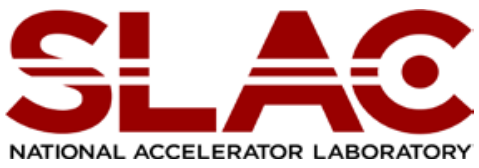




\section{A. Overview of the Current LCLS Facility}

LCLS uses the last one third of the SLAC linac for creation and manipulation of highly compressed electron bunches whose energy is partially converted into $x$-ray flashes of ultrashort duration and unprecedented brightness by a long undulator. Figure 1 provides a summary of the basic properties of LCLS as of March 2013.

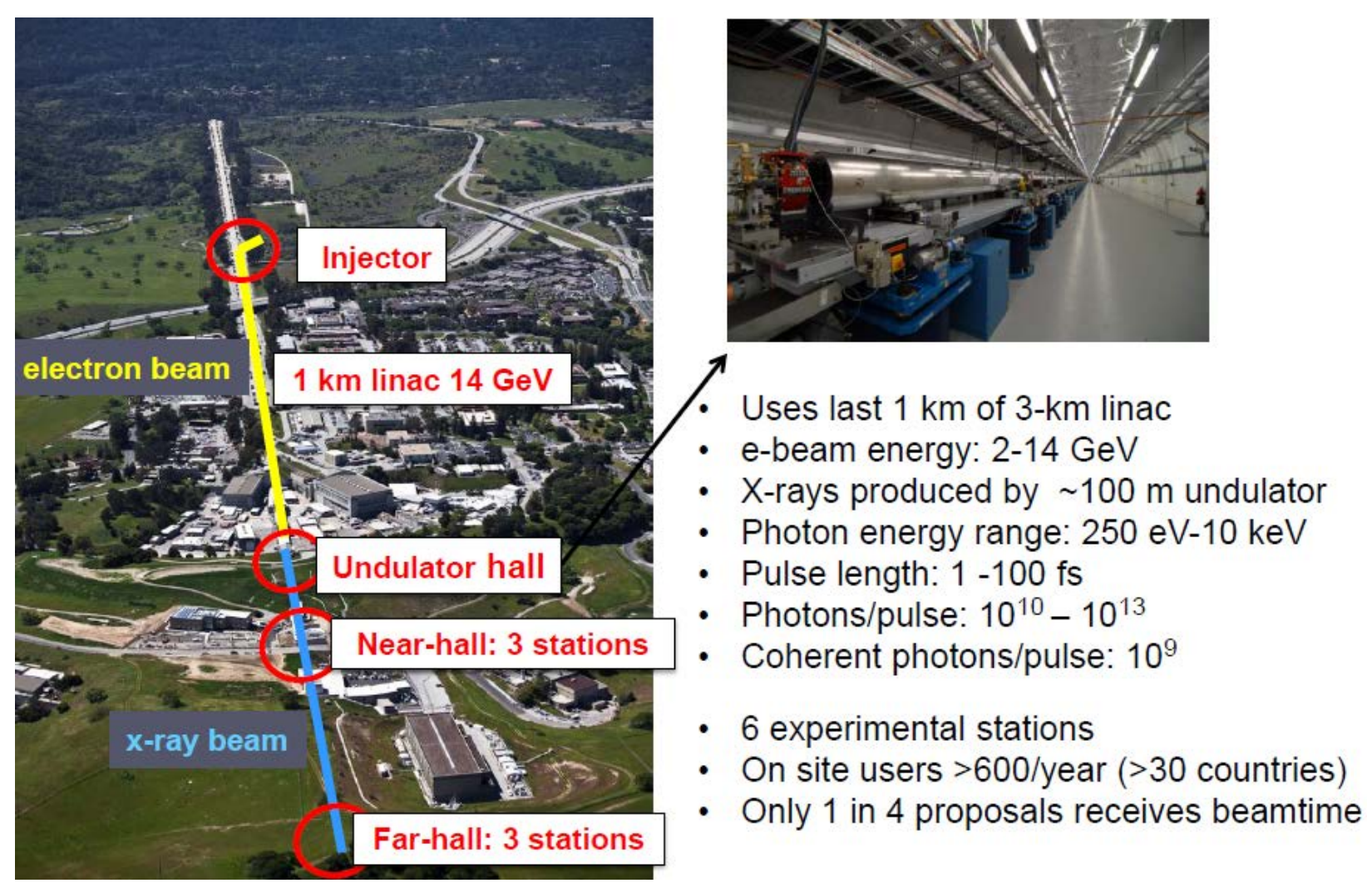

Figure 1: Aerial view of the SLAC and the linac (left) with overlaid positions of the key facility elements of LCLS. The picture on the top right shows the undulator hall and the key parameters of LCLS are listed underneath.

LCLS began its operations in October 2009 with user-assisted commissioning of the Atomic, Molecular and Optical (AMO) instrument. Since then, LCLS has rapidly evolved as a user facility and by now all six experimental stations support user science in areas summarized in Figure 2. 
Near Experimental Hall

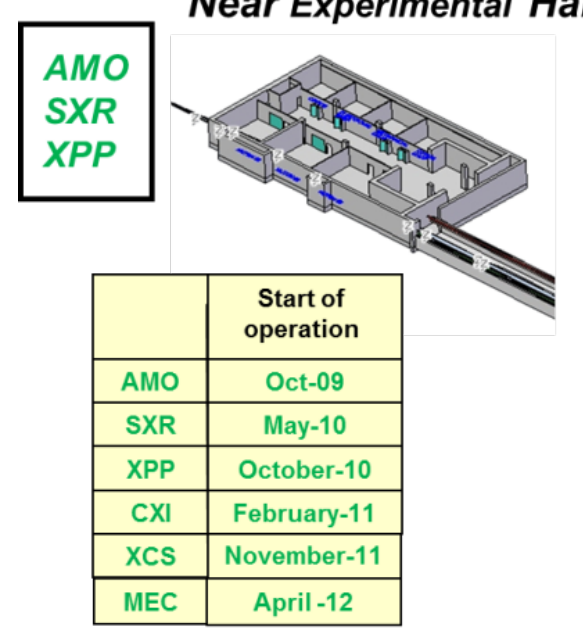

Far Experimental Hall

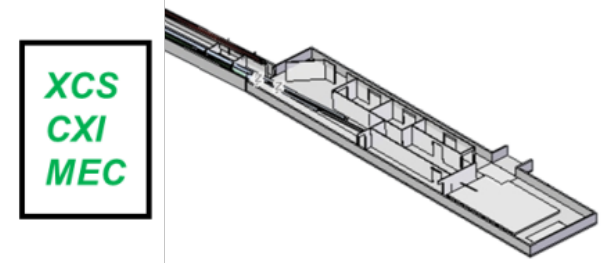

AMO: How do atoms and simple molecules respond to $\mathrm{x}$-ray laser light?

SXR: How do new electronic and magnetic properties emerge in complex materials and how do chemical reactions evolve at surfaces?

XPP: How do the atoms in materials and chemical complexes respond to excitations?

CXI: Can we solve the detailed atomic structure and function of bio-complexes that cannot be crystallized, are damaged by extended radiation exposure, or need to be in their natural state?

XCS: Can we capture the atomic motion in disordered materials and liquids as we change their equilibrium?

MEC: How do the properties of matter change when we take it far, far from equilibrium?

Figure 2: Overview of LCLS experimental stations and the science challenges they address.

\section{B. Five Year Research and Development Plan}

Research and development plans at LCLS are developed in close collaboration between accelerator, laser and $\mathrm{x}$-ray scientists. Projects are prioritized by the facility management in coordination with the Scientific Advisory Committee (SAC) and executed under close adherence to scope, schedule and cost. Here we present an overview of the LCLS R\&D programs with a five year horizon. Plans for the expansion of the LCLS facility, called LCLS-II, are given in section C. The overall strategic plan spans more than a decade of successive improvements cementing LCLS' leadership position in the US and worldwide.

LCLS supports a broad R\&D program in four general categories: e-beams, x-ray beams, conventional lasers, and end stations. $R \& D$ in e-beams and $x$-ray beams consist of beam generation, diagnostics and manipulation, conventional laser R\&D focuses on spectral range extension (to UV and $\mathrm{THz}$ ) and timing diagnostics, and end-station R\&D includes sample delivery, detectors and data analysis.

In the past, the LCLS R\&D program has produced notable outcomes that have been immediately implemented as part of the user program. Hard x-ray self-seeding was the first major milestone that yielded approximately a factor of 40-50 reduction in photon bandwidth and about two 
orders of magnitude improvements in photon energy stability relative to the non-seeded SASE beam. The second accomplishment was the development of pulse cross correlation techniques between optical and x-ray pulses. Pulse-by-pulse relative timing with an accuracy approaching $10 \mathrm{fs}$ is now available for pump-probe experiments. Most recently, there has been a successful demonstration of simultaneous $x$-ray pump-probe and nanocrystallography experiments through beam sharing using a thin diamond crystal large-offset double-crystal monochromator. This is now being implemented for increased user beam time.

\section{Accelerator and X-Ray R\&D}

Figure 3 provides an overview of LCLS R\&D projects with color coding indicating their present status. The blue colored projects are presently being pursued and will be finished and included in user operations within the next five years. The CATHO and XTCAV projects are respectively directed at improved cathode reliability for the LCLS rf photocathode gun and development of an $x$-band transverse cavity which is effectively an electron beam streak camera that will provide pulse by pulse information on the $\mathrm{x}$-ray temporal structures. Cross-correlation techniques are developed to precisely time the $x$-ray pulses relative to the optical pulses.

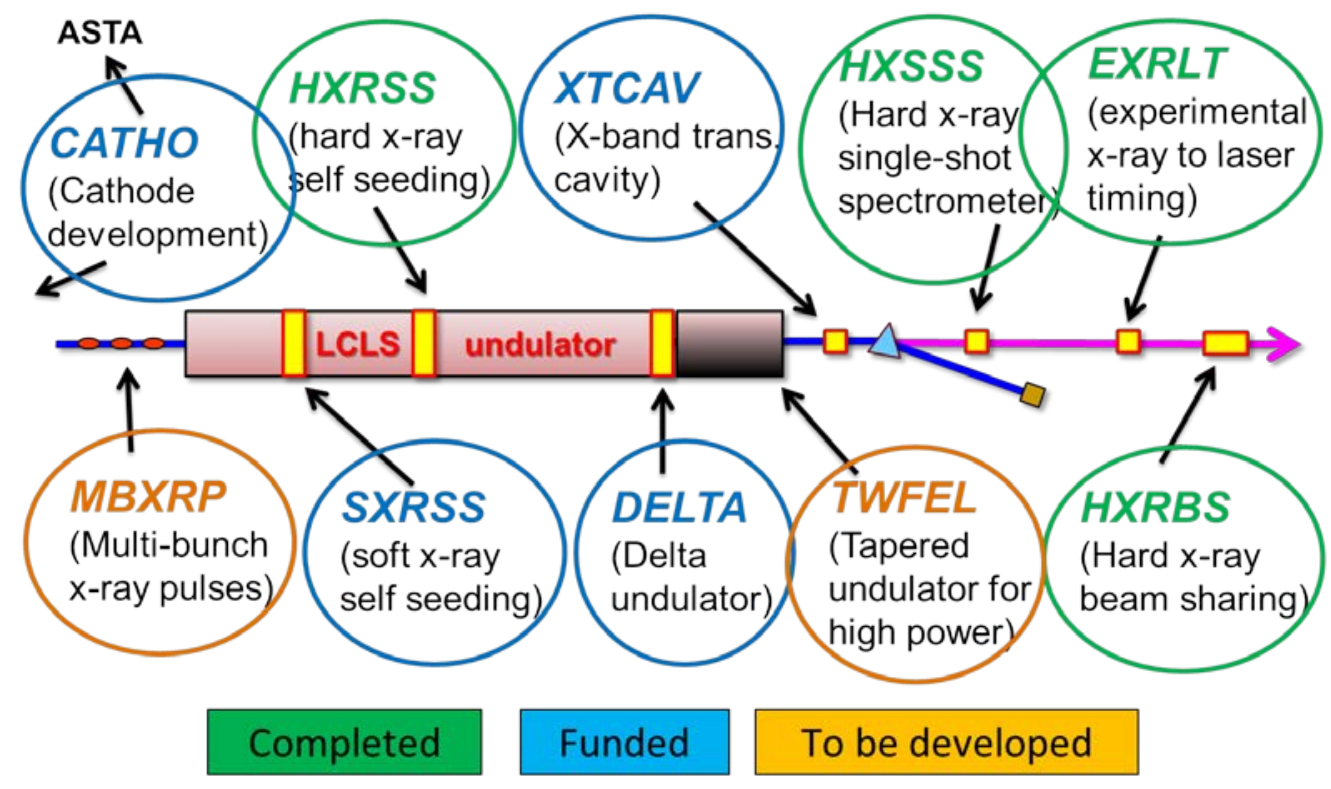

Figure 3: LCLS R\&D projects: completed projects are in green, funded and ongoing projects in blue, and future projects (to be developed) in orange.

A key area of future R\&D is the development of $x$-ray pulses with improved properties. While many first generation $x$-ray experiments took advantage of the unprecedented intensity and duration of X-ray laser pulses, user science increasingly demands improved control of the pulses, such as intensity stability, controlled pulse length, energy resolution, polarization, and 
the availability of multicolor pulses with variable delay. These developments clearly follow the developments of optical lasers over the last fifty years.

Figure 3 lists four R\&D projects aimed at the delivery of improved x-ray pulses. Already funded is the important SXRSS project providing seeded pulses. This project addresses improvements of the longitudinal phase space of the photon beam. Longitudinally coherent, known as transform limited, pulses minimize the uncertainty between pulse length and pulse energy. The goal is to create such pulses through hard and soft x-ray self-seeding and a new concept, iSASE or improved SASE, which is being explored without major investments during machine development studies at LCLS. The DELTA project is based on the production of circularly polarized soft $x$-rays by developing a novel DELTA undulator. Future plans include generation of multiple $x$-ray pulses through the MBXRP project and an ugrade to TW power by means of the TWFEL project. The latter project requires the availability of additional undulators and depending on implementation schedule and number of added undulators may require resources beyond those available through the LCLS operations budget.

\section{Schedule}

For the CATHO project a robust procedure will be developed in the recently upgraded ASTA facility at SLAC to restore or replace an LCLS gun cathode and obtain adequate quantum efficiency, thermal emittance and lifetime for reliable operation. The XTCAV project will be commissioned in FY13 to provide a shot-to-shot, non-invasive monitor of both the $x$-ray and ebeam temporal profiles with a few femtosecond resolution. Such information will be invaluable for interpretation of experimental results as well as for FEL physics developments.

In FY14, both the SXRSS and DELTA projects will be completed and integrated into user operation. Soft $x$-ray self-seeding will cover the photon energy range of 500-1000 eV (expandable to 300-1200 eV), which should improve photon bandwidth and peak brightness by about a factor of ten. In addition, a 3.2-m DELTA undulator is being prototyped to deliver arbitrarily polarized $x$-rays in the soft $x$-ray regime. In parallel with the development of the DELTA undulator, we are collaborating with X-FEL on $x$-ray diagnostic developments such as a shot-by-shot transmissive polarization analyzer for soft $\mathrm{x}$-rays.

In FY14-16, the R\&D efforts will focus on adding five undulators in the LCLS undulator hall to demonstrate the increased taper efficiency towards TW FEL. Simulations and preliminary machine studies show that the seeded FEL can benefit from stronger and longer tapered undulator. This will be a significant step for the future TW expansion of the LCLS-II undulator. Photon diagnostic development will also continue focusing on transmissive diagnostics for spectral analysis in the soft $\mathrm{x}$-ray regime and wavefront analysis for both soft and hard $\mathrm{x}$-rays to permit on-line optics alignment.

In FY 14-17, the R\&D efforts will also focus on developing multiple electron bunches within a macropulse. This development is critical for two bunch self-seeding and the production of multiple electron bunches that can be switched to different (LCLS-II) undulators to increase the 
rep. rate of each undulator. Efforts will also be directed on the development of x-ray optics for two color self-seeding, within the SASE bandwidth, in both the soft and hard x-ray regimes

\section{Laser R\&D}

LCLS R\&D on lasers focusses on two areas: Extension of spectra range and improved timing diagnostics.

LCLS presently has three sources, Difference Frequency Generation (DFG) to serve the 15-20 micron (20-15 THz) wavelength range, optical rectification in Lithium Niobate $\left(\mathrm{LiNbO}_{3}\right)$ for the 1-1.5 THz and Dimethl-amino-4-N-methylstilbazolium-tosylate (DAST) for 2-2.5 THz ranges. We have now demonstrated field strengths of $>1 \mathrm{MV} / \mathrm{cm}$ with the DAST. Future work is to improve conversion efficiency, bandwidth control, and wavelength tunability. Matthias Hoffmann has already shown that Cryo-cooling of the $\mathrm{LiNbO}_{3}$ can produce $3 x$ higher output, and temperature optimization is expected to yield even more efficiency. We will be investing considerable effort into making delivery of THz radiation to experiments more robust. The DAST is pumped in the mid-IR so it requires the OPA as well as all of the THz transport and diagnostics. Beam delivery, and most importantly focusing and overlap with the x-rays is extremely time demanding. We will be working on techniques to make this considerably easier.

We are also looking to extend the spectral range available to users to higher energies. We have already carried out one experiment with 20-40 eV High Harmonic Generation (HHG) pulses and are looking to extend this so that we can provide 10-100 eV light with control over spectral content. We are also pursuing techniques that will allow us to provide shorter pulses. In this case we are looking to provide sub-10fs pulses in the IR and the UV.

We have demonstrated <10fs timing in post processing which is desired by many users under the leadership of Ryan Coffee. However, making this available to general users requires significant support personnel consisting of laser and electronic control experts. We plan to develop robust engineering of the timing tool as part of our XIP program. R\&D efforts are required to determine $\mathrm{x}$-ray pulse shapes using pulse retrieval techniques. We have existing SBIR funding through Dan Kane of Mesa Photonics, the co-inventor of Frequency Resolved Optical Gating (FROG), to work with Ryan Coffee on pulse reconstruction techniques that could be used to measure the temporal profile of the X-ray pulses from LCLS.

Over FY2013-15 the MEC femtosecond laser system will undergo a two-step upgrade from 4 to 200 TW. This transformation will involve additional multi-pass and double-pass amplifiers, a new pump laser and the capability to utilize the MEC nanosecond laser system as a pump. This laser upgrade will enable new science at MEC: including investigations of hot electrons and energetic proton beams. This project is funded by the DOE Office of Fusion Energy Science (FES). 


\section{Detector R\&D}

Meeting the present and anticipated needs of LCLS I and LCLS II, respectively, requires the development of a new family of detectors. Not doing so, will compromise the science reach of LCLS. To minimize cost, risk, and schedule duration, all members of this detector family will share an overall architecture and the majority of their components. This strategy will allow a modest number of staff to efficiently originate a suite of detectors over the next five years. A system with small pixels and low noise for excellent single photon reconstruction is being designed and prototypes are expected in 2013. This low-noise detector will serve X-ray Photon Correlation Spectroscopy (XPCS), hard x-ray crystal-based spectrometers, and general imaging applications down to about $2 \mathrm{keV}$.

The next detector design is for increased count rate per pulse per pixel while maintaining single-photon sensitivity. This large-signal detector will be crucial for the Nanocrystal X-ray Diffraction instruments planned for LCLS-II. On the soft $\mathrm{x}$-ray side a general purpose area detector with single-photon discrimination down to the carbon edge is planned. For ARPES and other techniques SLAC intends to design a pixilated camera that provides high angular resolution and sub-nanosecond timing for soft electrons. Advanced packaging and interconnect technologies will be explored to minimize insensitive gaps in tiled sensor arrays. When appropriate collaborations academic and commercial institutions will be pursued.

\section{Sample Delivery R\&D}

Sophisticated sample delivery systems are commonplace at LCLS. These systems are used to deliver all manner of solid, liquid and gaseous targets such as protein nanocrystals, aerosol sprays of bio-matter and other systems, or fine liquid jets of solutions of molecular systems into the LCLS beam. In most instances these systems deliver samples into a vacuum environment, which adds to their complexity. In the past, these sample delivery techniques and systems have typically been developed by independent user groups. Although there has been early success with these systems, they remain rate limiting components during the course of an experiment and reduce the overall efficiency of LCLS operation. In addition, not all systems are available to the general user community since they are user developed and provided. To address these issues, LCLS has created a dedicated sample delivery department, headed by Daniel DePonte. As a world leader in the aforementioned systems, Dr. DePonte is responsible for leading the $R \& D$ effort associated with developing robust, cutting-edge sample delivery techniques and systems. 


\section{Long Range Strategic Plan}

The long range LCLS strategic plan outlined below is designed to keep LCLS internationally competitive and provide increased user capacity and capabilities. It builds on the utilization of the present six LCLS instruments and outlines the envisioned evolution of LCLS through 2025. Key elements of this strategic plan are briefly outlined in this section. More details can be found in the whitepaper "Science Driven Instrumentation for LCLS-II" on the LCLS website.

The strategic evolution of the core accelerator and x-ray instrumentation capabilities over the next $10+$ years is schematically illustrated in Figure 4 . The next step in the expansion of the current LCLS facility (hereafter referred to as LCLS-I) is delivery of the LCLS-II baseline project (CD0 in April 2010), with first light in 2018. LCLS-II provides a second injector and another $1 \mathrm{~km}$ of linac which operate independently from LCLS-I and by means of two new undulators deliver soft and hard $x$-rays to a new experimental hall containing one experimental station. We are presently preparing a proposal for the next step, a fully instrumented LCLS-II facility. Key elements of this development are the addition of new soft and hard x-ray source capabilities such as seeding, polarization control and increased $x$-ray power, and the construction of up to six additional $x$-ray instruments in the LCLS-II experimental hall. The final step, labeled LCLS2025 consists of adding another hard x-ray undulator into the LCLS-I tunnel and adding two more hard $x$-ray instruments in the first LCLS-I experimental hall.

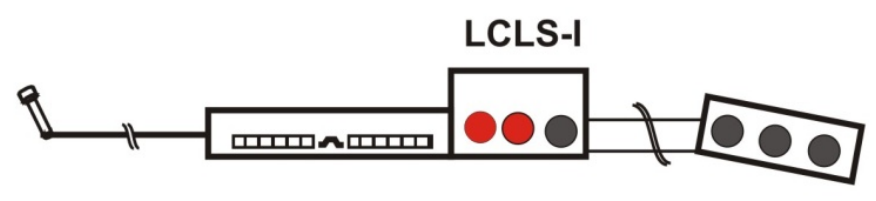

LCLS-II baseline

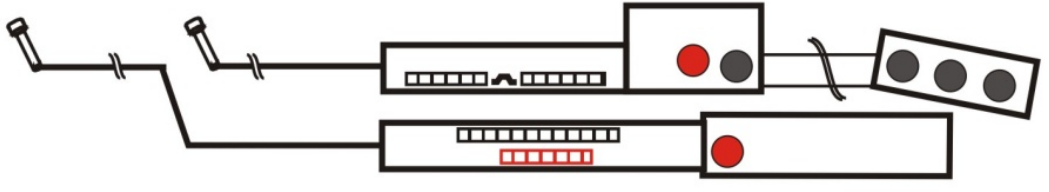

LCLS-II fully instrumented
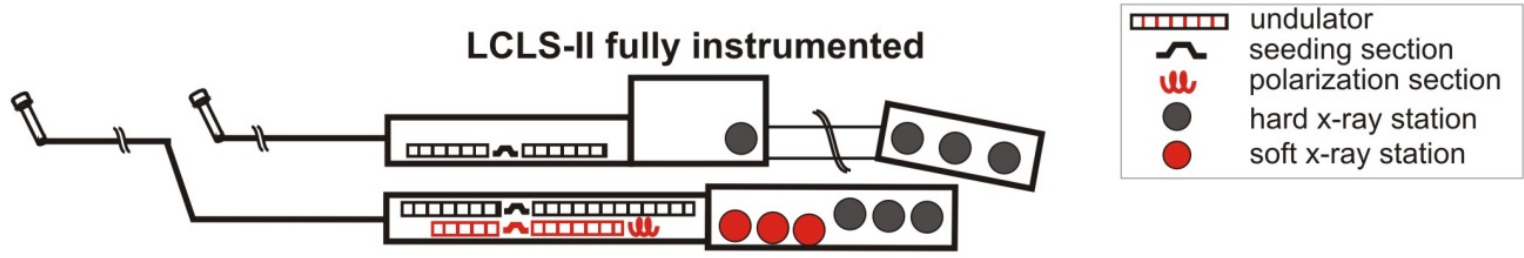

LCLS 2025

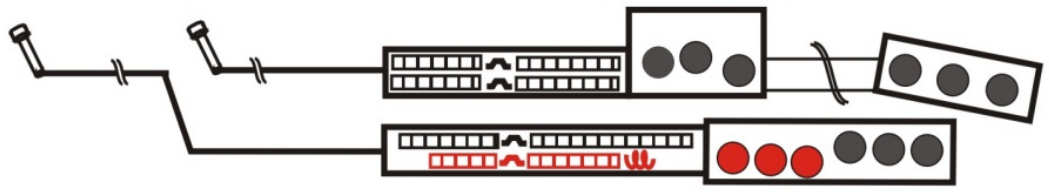

Figure 4: Key expansion steps of LCLS with soft $\mathrm{x}$-ray undulators and stations shown in red and hard $\mathrm{x}$ ray capabilities in black. Descriptions of the icons are given on the right. 
The key enhancements of a fully instrumented LCLS-II over the current LCLS-I facility are:

- Two independent injectors for flexible x-ray pulses

- Tripling of the number of undulator sources

- Tripling of the simultaneously operating stations

- Decoupling of hard and soft x-ray operation

- Extended photon energy range to lower and higher energies (250eV-18keV)

- Self-seeded, near transform limited soft and hard x-ray pulses

- Pulse enhancements of 1000X in brightness, 10X in power and 100X smaller bandwidth

- Complete soft x-ray polarization control

- X-ray pulse manipulation (split, delay, combine, multi-colors)

- Improved $x$-ray detectors (single photon sensitivity, dynamic range, number and size of pixels, $120 \mathrm{~Hz}$ for soft x-rays)

- Pump-probe timing to 10 femtoseconds

Figure 5 illustrates the LCLS capacity growth in terms of the number of undulator sources and experimental stations for the four steps in Figure 4.

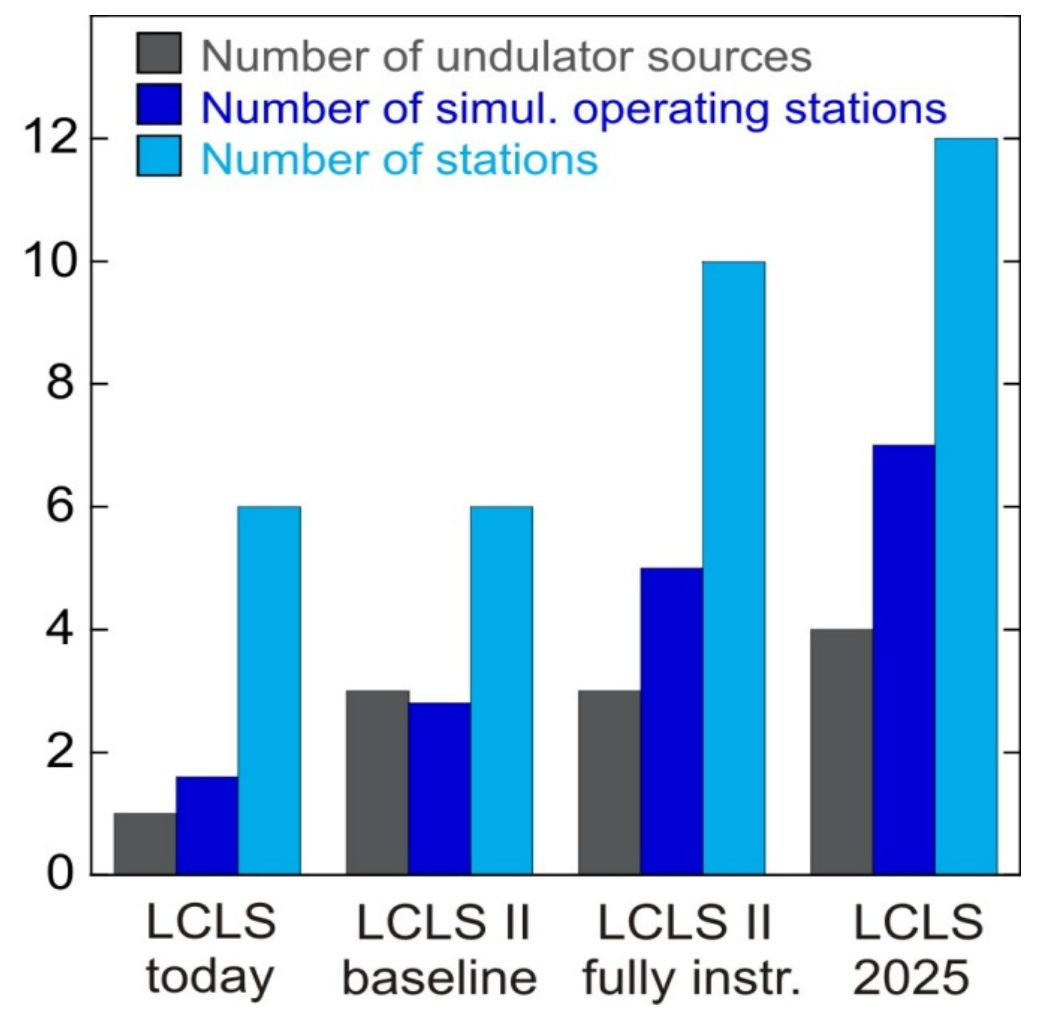

Figure 5: LCLS growth in number of undulators and stations for the four scenarios shown in Fig. 4. Fractional numbers indicate that not all stations can operate simultaneously. 
A fully instrumented LCLS-II will enhance user science in all science areas of LCLS-I outlined in Figure 2. Owing to enhanced soft x-ray capabilities such as self seeding, AMO science will benefit from enhanced power densities at LCLS-II and a new AMO station is being constructed as part of the core LCLS-II project.

A future MIE is envisioned to support the construction of new stations for three key science areas: novel materials, chemical reactivity and biological structure. The scientific case is elaborated in the strategic plan document referenced above and the link between these science areas, their major impact areas, and the required instrumentation is summarized in Figure 6 . We note that the MEC program will also benefit from the advent of LCLS-II since more hard x-ray time will become available for the four remaining instruments in the LCLS-I experimental halls as shown in Figure 4.

Below we briefly outline the scientific drivers for a fully instrumented LCLS-II, following Figure 6.

$\begin{array}{lll}\text { Emergent Phenomena in } & \text { Chemical Reactivity in } & \text { Structure of Protein } \\ \text { Advanced Materials } & \text { Nature and by Design } & \text { Nanocrystals } \\ \text { Information Technology } & \text { Energy, Environment } & \text { Drug Design, Health }\end{array}$

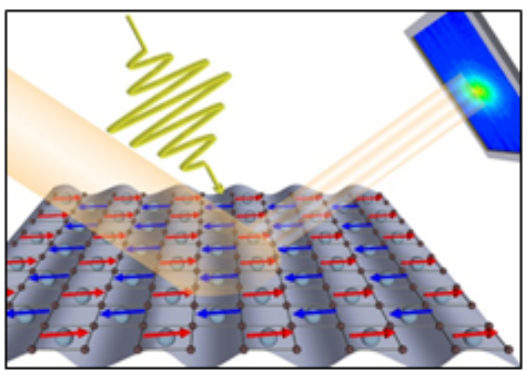

Atoms/Electrons/Spins

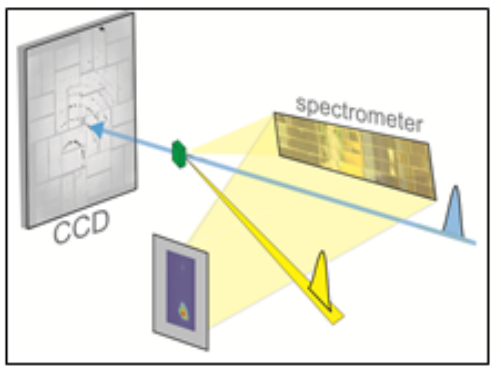

Atoms/Electrons

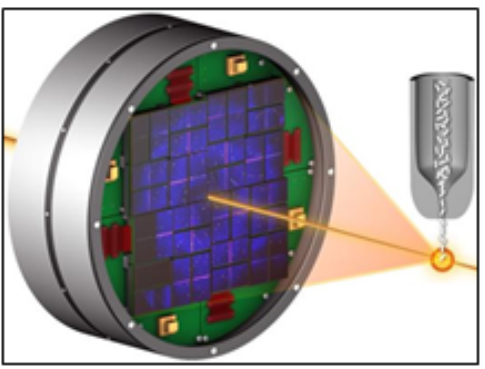

Atoms

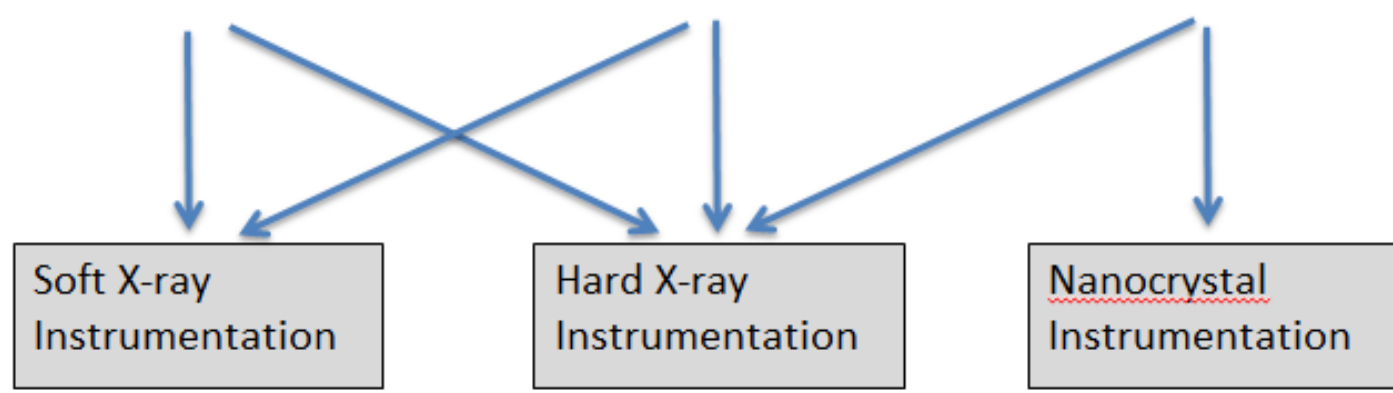

Figure 6: Schematic of three high priority science and impact areas for LCLS-II, and their coupling to selected instrumentation, as described in the text. 


\section{Understanding and Controlling the Complex Interplay between Distinct Orders in Quantum Materials}

A key challenge in modern condensed matter physics is the understanding of emergence of exotic quantum phases that can be controlled by chemical compositions and applied fields and therefore are envisioned to extend today's "silicon and metal" technology. These materials exhibit useful properties such as high temperature superconductivity, colossal magnetoresistance and multiferroicity that emerge from charge/spin/orbital ordering in electronically distinct domains with temporal and spatial fluctuations over many time and length scales. LCLS-II will facilitate detailed studies of these emergent phenomena in the critical energy domain of $\mathrm{meV}$, spatial domain of nanometers and temporal domain of femtoseconds to picoseconds. Of key importance will be the availability of well controlled pulses of variable polarization, pulse length and energy resolution combined with novel instrumentation and techniques, such as time-resolved Resonant Inelastic X-Ray Scattering (RIXS) with possible signal enhancement through stimulated processes. The ultimate goals are understanding the underlying organizing principle of the emergent phenomena, and controlling them and properties of these novel quantum phases.

\section{Controlling Photochemical Transformations on the Molecular Level}

One of the grandest scientific challenges with large impact on quality of human life is the understanding and control of chemical reactivity and transformations at the molecular level. Such processes often occur extremely fast at local catalytic reaction centers thus requiring probing methods with very high spatial and time resolution as well as chemical specificity beyond what is currently available with SR sources. Controlling chemical reactivity promises the synthesis of many new useful products, efficient and compact energy storage and release by chemical bond manipulation, and the ability to influence key biological reactions and functions. A prime target for LCLS studies are reactions triggered by optical photons. They have been utilized by nature and can now guide us in the development of artificial processes that evolve with enhanced efficiency. Ultrafast optical pulses can trigger these processes whose evolution is then probed with well-timed and controlled $x$-ray pulses provided by LCLS-II. The subsequent atomic motion can be tracked by ultrafast crystallography and diffuse hard $\mathrm{x}$-ray scattering techniques. Soft $\mathrm{x}$-ray spectroscopies will allow the observation of local changes in bonding around selected atoms and in selected functional groups. Since these local changes may involve very subtle bonding changes, conventional techniques will have to be complemented by higher sensitivity non-linear techniques, such as stimulated soft $\mathrm{x}$-ray Raman scattering. Such studies require the use of transform limited $x$-ray pulses of different color and polarization provided by LCLS-II.

\section{De-Novo Structure Determination of Challenging Biological Targets}

Determination of the complex structure of African sleeping sickness has been recognized by Science magazine as one of the nine runners-up to its selected science "Breakthrough of the 
Year". The Science article states that the success at LCLS "shows the potential of X-ray lasers to decipher proteins that conventional X-ray sources cannot." Solving radiation-damage-free structures of the most challenging and important proteins and complexes which cannot be solved by SR based methods will require independent, de-novo structure determination using single wavelength anomalous diffraction (SAD) and multiple wavelength anomalous diffraction (MAD) techniques with highly accurate spectral intensity and pulse control. In many cases x-ray energies above $10 \mathrm{keV}$ are needed as they are critical for highest resolution and important edges for phasing such as Se and Br. Well controlled photon pulses for most accurate spectral intensity measurements with a maximum number of fully recorded reflections will minimize the need for oversampling, an important limitation for proteins that are difficult to prepare and crystallize. The LCLS-II hard $x$-ray instrumentation will provide these capabilities. High intensities, such as those produced by LCLS-II soft x-ray instrumentation, are needed to provide enough signal to image structures of non-periodic biological objects such as viruses with single shots, albeit at lower resolution. A variety of wavelengths and focal spots will become available to provide the imaging tools required to study a wide range of biological systems, and LCLS-II will extend the current soft x-ray capabilities of LCLS to 2,500 eV.

\section{Operational Efficiencies}

Over the first four years of user operation, LCLS performance has exceeded all expectations. LCLS now operates with $95 \%$ of scheduled beam delivery and today's beam capabilities greatly exceed those of the baseline project. The x-ray energy per pulse has been increased from $<2$ $\mathrm{mJ} / \mathrm{pulse}$ to a maximum of $6 \mathrm{~mJ} / \mathrm{pulse}$. The energy range has been extended from $800 \mathrm{eV}$ down to $250 \mathrm{eV}$ (although spectroscopy near the C K-edge $(285 \mathrm{eV})$ is still limited by reduced reflectivity of the boron carbide mirrors) and from $8 \mathrm{keV}$ up to $10 \mathrm{keV}$. The pulse length has been shortened from the nominal $230 \mathrm{fs}$ down to $\sim 1 \mathrm{fs}$. Pulse timing between $x$-ray and optical pulses has improved from 200 fs to $10 \mathrm{fs}$. The original Self Amplified Spontaneous Emission (SASE) pulse production has now been complemented with self-seeded hard $\mathrm{x}$-ray pulses that are nearly transform-limited. Such completely laterally and longitudinally coherent pulses are the hallmark of a true laser and enable the extension of $\mathrm{x}$-ray science to non-linear $\mathrm{x}$-ray science with controlled pulses. Most recently, beam sharing of hard x-rays by two experimental stations has been successfully demonstrated by use of a thin diamond crystal that transmits about $70 \%$ of the beam to one station while reflecting a monochromatic slice into another station. This capability will significantly increase scheduled user time in the near future.

The evolution of LCLS operations has been accompanied by a strong growth in personnel associated with x-ray user operations and a reduction in accelerator related personnel as illustrated in Figure 7 and discussed in the following two sections.

\section{Accelerator Operational Efficiencies}


The Accelerator Directorate has undergone significant organizational changes during the past two years. A major re-organization took place to flatten the organization and reduce the overhead required to operate all divisions. In addition, a number of service groups reduced support from a 24/7 staffing to call-in support. This was possible by evaluating the cost/benefit ratio of the provided support and implementing remote control systems. An example of successfully streamlining the organization is the involvement of the accelerator operators in personnel protection system checks and certifications, which previously were solely carried out by the Safety Systems department of the Instrumentation and Controls Division.

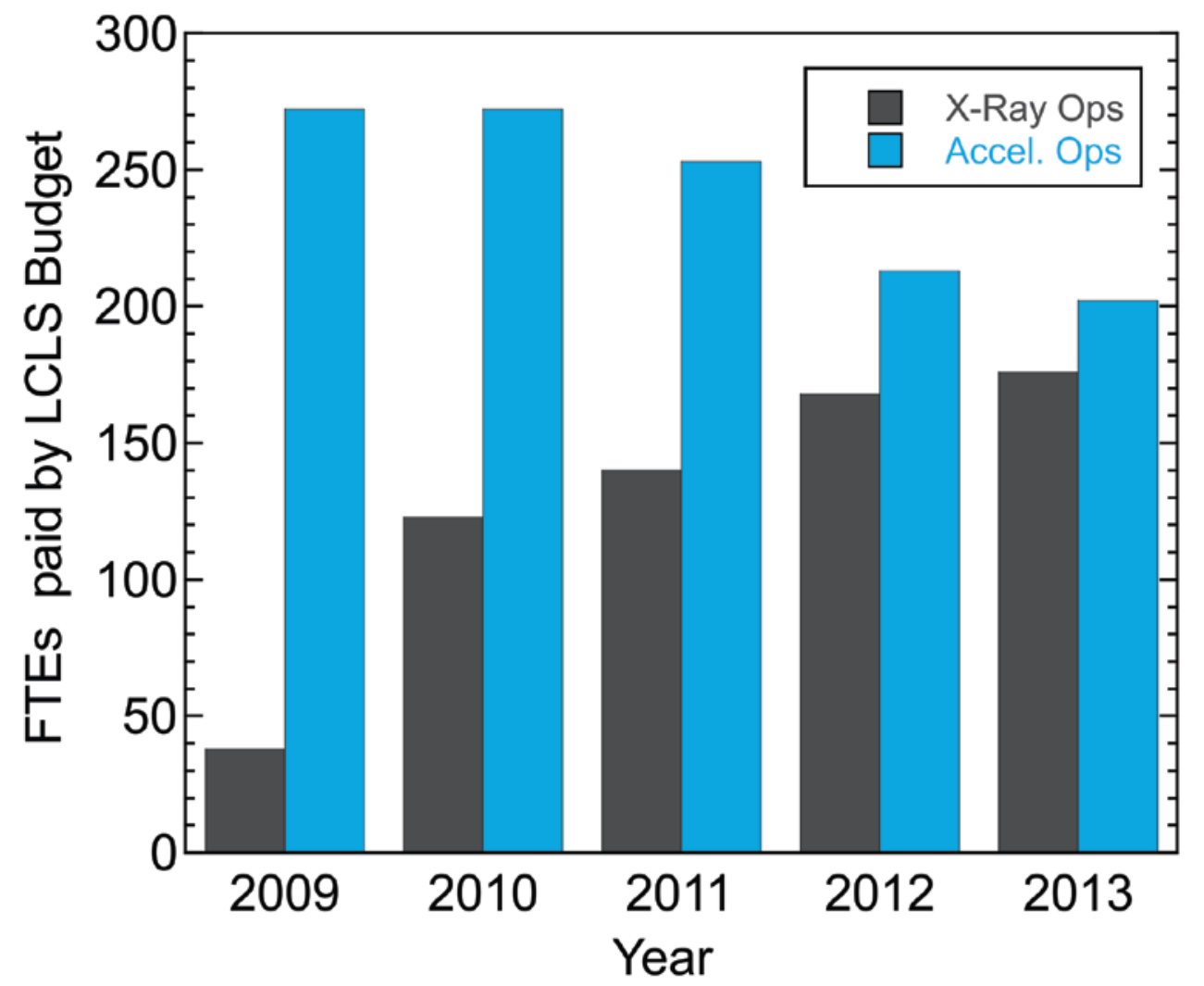

Figure 7: Number of FTEs funded by the LCLS-I ops budget in two major categories as a function of time.

Further optimizations include the consolidation of machine shops throughout the site, evaluating "make versus buy" decisions on a broad scale and supplementing the permanent work force with contractor supplied - field managed part time work force especially through shut downs or other peak work load periods of time.

Over the last two years a staff reduction of $20 \%$ has occurred from a total of 253 to 202 FTEs. The downsizing of the accelerator related workforce was accompanied by an approximately $\$ 10$ million decrease of the accelerator related budget from $\$ 80$ million in FY11 to $\$ 70$ million in FY13 while maintaining / growing the R\&D investments. This was also required to support the 
rapid growth in x-ray related personnel necessitated by the adding of user end stations, and increased laser and detector support. Going forward we only envision optimizations of accelerator staff within the LCLS-I operations budget.

Growth in staffing and operational expenditures will however be needed during transition to LCLS-II operations. Compared to the current effort, the number of operating accelerator and beam systems will more than double. In addition to the added 10 accelerator sectors, we will operate at least two additional beam transport and undulator systems, as well as the electron beam systems required for transport of the beam from the end of the LCLS-II accelerator (S20) to the new undulator hall. We anticipate taking advantage of economies of scale so that the total operation of LCLS-I plus LCLS-II systems can be accomplished by a 30 percent increase in operating expenses. A more detailed analysis of the transition to LCLS-II is given in section 3. III. In addition a mission readiness assessment has been performed for all accelerator related components and the accelerators itself. With many systems coming close to their end of life, a flexible investment strategy has been developed which would allow further reduction for LCLS (SSRL) support and very limited growth in staff with LCLS II coming online.

\section{X-Ray Operational Efficiencies}

The user experiments performed at LCLS are typically first of their kind and complex. The complexity is primarily due to the nature of the $x$-ray free electron laser source that exhibits significant shot-to-shot fluctuations in almost every pulse parameter (profile, position, spectrum, timing, etc.). The experimentalists must address these fluctuations to perform a successful experiment. Multiple "state-of- the-art" systems are required to tackle the challenges presented by the LCLS:

- Custom x-ray area detectors capable of recording individual pulses at $120 \mathrm{~Hz}$

- Sophisticated diagnostics that measure the pulse properties on a shot-by-shot basis (timing, spectrum, intensity)

- Data acquisition systems capable of merging the data acquired by various detectors with rates reaching $2 \mathrm{~GB} / \mathrm{s}$

- Data storage systems that can accommodate $~ 50$ TB of data per experiment

- Data analysis systems that can efficiently filter and sort the data based on measured perpulse parameters

- Flexible ultrafast laser systems that are able to tune their wavelength to pump specified degrees of freedom (mid-IR to UV, THz)

- Femtosecond timing systems used to synchronize the optical pump lasers

In order to maximize the scientific output, LCLS management has implemented a user support model that provides a very high level of service. In particular, four scientific staff are assigned to each user experiment. This level of support permits 24-hour coverage for both the days leading up to the experiment and the days during the experiment execution. We believe this model has 
been the key to the early success of LCLS (high experiment success rate and high level of user satisfaction).

In the future, the amount of beamtime available at the LCLS complex will more than triple to do the construction of LCLS-II and via beam multiplexing efforts. Our goal is to maintain the same level of user support but with a reduced staffing team - allocating two scientific staff per experiment rather than four. We aim to realize this gain in efficiency by a combination of the following:

- Simplifications to our subsystems and user interfaces

- Cross training our scientific staff to

- Training of LCLS users

- Construction of dedicated experimental setups

The realization of this gain in efficiency will require an extensive effort and will likely span multiple years. However, we believe it will be implemented in time before the start-up of LCLSII operations. 